\title{
Stability of behavioral syndromes but plasticity in individual behavior: consequences for rockfish stock enhancement
}

\author{
Jonathan S. F. Lee • Barry A. Bereijikian
}

Received: 7 April 2007 / Accepted: 5 August 2007

(C) Springer Science + Business Media B.V. 2007

\begin{abstract}
This study investigated behavioral syndromes, which are defined as correlations between behaviors. Behavioral syndromes can lead to the unintentional alteration of a wide range of behavioral traits of hatchery fish if unintentional selection on one behavior leads to selection on a correlated behavior. Specifically, this study used brown rockfish, Sebastes auriculatus, to test the hypothesis that a fish that feeds at high rates in the absence of a predator also takes more risks when a predator is present, and that through such a correlation, unintentional hatchery selection for high feeding rates may also lead to changes in risk taking behavior (here defined as behavior that increases predation risk). Behavioral syndromes were found-feeding behavior in the absence of a predator tended to correlate positively with both feeding behavior in the presence of a predator model and time near the model. These syndromes were stable through time - that is, the same correlations appeared 10 days
\end{abstract}

J. S. F. Lee $(\bowtie)$

Department of Neurobiology and Behavior, Cornell University,

Ithaca, NY, USA

e-mail: jon.lee@noaa.gov

Present address:

J. S. F. Lee · B. A. Bereijikian

Manchester Research Station,

Resource Enhancement and Utilization Technologies

Division, Northwest Fisheries Science Center, NOAA,

P.O. Box 130, Port Orchard, WA 98353, USA later when the behavioral assays were repeated. However individual behavior was inconsistent (plastic). A fish could both feed and take risks at high rates on Day 1, but then both feed and take risks at low rates on Day 10. Thus, while behavioral syndromes were stable (i.e. present in both rounds 1 and 2), individuals were plastic in their behavior (i.e. inconsistent between rounds 1 and 2). After 16 weeks of hatchery rearing, neither growth nor survival were predicted by behavior. It is suggested that the behavioral plasticity within individuals through time makes consistent selection for strong feeders less likely, and that species with more plastic behavior may be less susceptible to unintentional selection on behavioral syndromes than species with behavior that is more fixed.

Keywords Hatchery - Unintentional selection · Behavioral syndrome $\cdot$ Sebastes auriculatus

\section{Introduction}

Stock enhancement seeks to aid the recovery of depleted fish populations through the release of cultured (hatchery) fish into the wild (Bell et al. 2006). However, the results of stock enhancement releases have been mixed (Molony et al. 2003), largely due to several problematic issues (Brown and Day 2002; Salvanes and Braithwaite 2006). For example, the supplementation of wild stocks can be economically inefficient if the hatchery environment leads to 
genetic or environmental effects that cause fish to suffer reduced fitness after release (Reisenbichler and Rubin 1999; Fleming and Petersson 2001). Also, if released fish differ genetically from wild stocks (Einum and Fleming 1997; Fleming and Einum 1997), and if hatchery and wild fish intermingle, then adaptive gene frequencies of wild populations could be altered (Lynch and O'Hely 2001).

Huntingford (2004, 2005) proposed a scenario through which the hatchery environment can produce individuals that behave maladaptively in the wild. In this scenario (Huntingford 2004), there exists a behavioral syndrome for feeding behavior. A behavioral syndrome is defined as a correlation between behaviors (Sih et al. 2004; Bell 2007; the terms "behavioral syndrome" and "behavioral correlation" will be used synonymously in this paper). That is, individuals that feed voraciously in the absence of a predator also feed voraciously in a different situation, such as when a predator is present. In contrast, other individuals in the same population feed more cautiously in both situations. If the predator-free hatchery environment selects for individuals that feed at high rates in the absence of predators, then through the behavioral syndrome, there may also be inadvertent selection for individuals that feed at high rates in the presence of predators ("risky" feeding). This mechanism would occur if selection on one component of a correlation effectively induces selection on correlated traits. Since animals often reduce feeding rates when exposed to a predator (Morgan and Colgan 1987; Lima and Dill 1990; Bishop and Brown 1992), it is reasonable to predict that the increase in "risky" feeding behavior may lead to high mortality rates of released fish. Further, if the variation is gene-based, gene frequencies in the wild could be altered. Thus, it is important to understand the factors that influence behavioral variation, and understand whether differential survival among variants occurs in hatcheries that release fish into the wild (Huntingford and Adams 2005).

If a behavioral syndrome exists, the temporal stability of both behavioral syndromes and of individual behavior may be important determinants of the strength of selection on the syndrome. The study of behavioral syndromes has increased markedly over the past several years (e.g. Duckworth 2006; Salonen and Peuhkuri 2006; Cote and Clobert 2007; Magnhagen 2007), but there have been few studies that investigate both the stability/instability of behavioral syndromes and the stability/instability of individual behavior. Bell and Stamps (2004) discussed these distinctions, and used sticklebacks to illustrate how behavioral syndromes can be unstable through time in some instances, and how stable behavioral syndromes can coexist with unstable individual behavior in other instances.

If a behavioral syndrome is unstable-for example present at one point in time, but not present at a later point in time-then selection on the behavioral syndrome might be weak since selection would be restricted to the time frame in which the correlation occurs. However if the behavioral syndrome is stable through time, but if individual behavior is plastic (e.g. at time $X$, a particular individual feeds at low rates in both the absence and presence of a predator, but then at time $Y$, feeds at high rates in both scenarios), then selection might also be weak since the behavioral plasticity may make consistent selection less likely. Thus, knowing the stability of both the behavioral syndromes and individual behavior may be important if we are to predict whether selection on a behavioral syndrome is going to occur.

Rockfish populations have been declining in the Puget Sound, WA and elsewhere (Parker et al. 2000; Levin et al. 2006); currently, investigators are developing methods for the stock enhancement of these fishes (e.g. Nakagawa et al. 2006). Little is known about the behavior of juvenile rockfish (Love et al. 2002). This study sought to increase our understanding of possible mechanisms for behavioral change in hatcheries, in the hopes that such knowledge will help hatcheries produce individuals that are more similar to wild individuals, and may ultimately aid in the recovery of depleted populations.

There were four objectives. The first objective was to determine the role that body size plays in generating variation in risk taking behavior (i.e. behavior that increases predation risk) in brown rockfish. To address this, feeding trials were conducted with small and large juvenile rockfish, under different levels of predation risk. Second, to document behavioral variation while minimizing the effect of body size differences, and to determine whether correlations existed among behaviors, the same experiment was conducted but with a restricted range of body sizes. Third, to test the stability of behavioral syndromes and of individual behavior through time, the above 
experiment was repeated with the same individuals; i.e. the same marked individuals were run through a second behavioral trial that was identical to the first. Finally, to determine whether the hatchery environment selects for certain behavioral phenotypes, these marked individuals were raised under typical hatchery conditions and evaluated to determine whether behavioral phenotype predicted growth or survival.

\section{Materials and methods}

The Seattle Aquarium obtained rockfish from field collections, and provided us with a pregnant female. Offspring were fed rotifers, Brachionus, brine shrimp, Artemia, and various wild zooplankton, and raised in $9.3 \mathrm{~m}^{3}$ bags off a pier in the Puget Sound through metamorphosis, before being weaned onto commercial salmon diet (Biodiet) and transferred to shorebased tanks.

The juvenile brown rockfish used in experiments were transferred from the shore-based tanks to 2081 aquaria at a density of approximately one fish per 81 . Water to holding and experimental tanks (see below) was supplied by a flow-through system that brought filtered water from the Puget Sound. Fish were kept on a 12:12 light-dark cycle and maintained on newly-hatched brine shrimp. The juveniles were approximately 15 months old at the time of the first experiment.

\section{Experiment 1 -predator model}

This experiment evaluated whether the rockfish perceived the sculpin predator model as a threat. The 14-cm plastic resin sculpin model was sculpted and painted by a taxidermist, based on a preserved sculpin, Cottus spp. Clear fishing line centered two wire rings around the model's mouth and delineated a 5 and a $10 \mathrm{~cm}$ radius from the mouth. More clear fishing line was attached to the model so that it could be gently lowered it into aquaria. At 1,500 h on Day 1, single rockfish ( $N=16$ fish, size range: 2.8 to $3.6 \mathrm{~cm} \mathrm{SL}$ ) were each placed in ten-gallon aquaria that contained a T-shaped PVC pipe for shelter and a horizontal PVC pipe that served as a siphon for the flow-through system. Food was delivered individually to each tank through access points in the flow-through system. Fifty milliliters of newly-hatched brine shrimp were added to each tank on Days 3 and 5.
On Day 7, rockfish were observed and several behaviors were recorded. A "dart" was defined as a rapid (less than $1 \mathrm{~s}$ ) forward movement of at least three body lengths. A rockfish's feeding (hereafter, "bite") on brine shrimp involves the opening of the mouth and a small forward movement (less than one body length), and was easily recognizable. Fifty milliliters of brine shrimp were added to each tank. After the first bite, the fish were allowed to feed for $200 \mathrm{~s}$ (Period 1). For the following $200 \mathrm{~s}$ (Period 2), either (a) the sculpin model was lowered into the tank (Predator treatment), the number of bites and darts were counted, and the number of seconds spent in the $5 \mathrm{~cm}$ radius and $10 \mathrm{~cm}$ radius ring near the sculpin were recorded, or (b) nothing was added (Blank treatment) and the number of bites and darts were recorded. In all experiments, the rockfish spent very little time within the inner ring, so the times in the inner and outer rings were combined for all analyses. Period 3 also lasted for $200 \mathrm{~s}$ and was identical to Period 2, except a fish that received the Predator treatment in Period 2 received the Blank treatment in Period 3, and vice versa. Treatment order was alternated between individuals. Fish used in this experiment were not used in any other experiment. To determine whether the rockfish perceived the sculpin model as a threat, a mixed model was used to test for differences in the number of darts and the number of bites at brine shrimp between the Predator treatment time period and the Blank treatment time period. The treatment was set as the fixed effect; the individual was the random effect.

Experiment 2-body size and behavioral variation

This experiment tested whether body size influenced feeding behavior and time near the predator model. At $1,500 \mathrm{~h}$ on Day 1 , single rockfish $(N=16$ fish, eight small: 1.7 to $2.6 \mathrm{~cm}$ SL and eight large: 2.9 to $3.5 \mathrm{~cm}$ SL) were each placed in 10-gallon aquaria that were identical to those used in the "predator model" experiment, and fed $50 \mathrm{ml}$ of newly hatched brine shrimp. On Day 3, another $50 \mathrm{ml}$ of brine shrimp was added to each tank. After the first bite, the number of bites in the next $200 \mathrm{~s}$ was recorded. After the $200 \mathrm{~s}$, the predator model was lowered into the tank. For the next $300 \mathrm{~s}$, the number of bites on brine shrimp and the amount of time spent in the wire rings were recorded. Fish used in this experiment were not used 
in any other experiment. The Mann-Whitney test was used to test for behavioral differences between small and large fish.

\section{Experiment $3 \mathrm{a}$ - behavioral variation, round 1}

The methods used in the body size range experiment (above) were followed, except with a narrower body size range $(N=41$ fish, range in round $1=3.0$ to $3.3 \mathrm{~cm}$; range in round $2=3.1$ to $3.4 \mathrm{~cm}$ ). After the experiment, fish were injected with unique color combinations of acrylic paint (Northwest Marine Technology, Tumwater, Washington, USA), and returned to holding tanks. These marks were still visible at the completion of this study.

Experiment $3 \mathrm{~b}$ - behavioral variation, round 2

After 8 to 12 days, Experiment 3 a was repeated using the same methods and fish from Experiment 3a. Fish were measured at the completion of the experiment. Analyses tested for correlations between time to the first bite, or the number of bites taken in the Prepredator treatment period, and the number of bites taken in the Predator treatment period, or the amount of time spent in the ring surrounding the predator. To gain an understanding of the temporal stability of individual behaviors, analyses tested for correlations in single behaviors between rounds 1 and 2 (e.g. correlation between Pre-predator bites in round 1 and Pre-predator bites in round 2).

Experiment 3c-long-term growth and survival

After Experiment 3b, the fish were transferred to rearing tanks where they were held at $8-12^{\circ} \mathrm{C}$ and fed once a day, 5 days per week, with dry commercial salmon diet. To determine whether individuals with different behavioral tendencies survived at different rates, the size and identities of the fish that were still alive 16 weeks after the completion of round 2 were recorded. The behavioral parameters from rounds 1 and 2 were averaged; analyses tested for differences in those averages between survivors and non-survivors. To determine whether individuals with different behavioral tendencies grew at different rates, analyses tested for correlations between the behavioral averages and growth, calculated as the difference in standard length between the beginning and end of the experiment. Analyses also tested for a correlation between size at the beginning of the experiment and size at the end of the experiment.

\section{Results}

Experiment 1-predator model

None of the 16 fish used in Experiment 1 darted in the Blank treatment, but seven of them darted in the Predator treatment. Thus, rockfish darted significantly more during the Predator treatment than during the Blank treatment (mixed model: $N=16, F=0.025$, $P<0.03$; number of darts in the Predator treatment, $X \pm \mathrm{SE}: 0.88 \pm 0.35$ ). Rockfish took significantly more bites during the Blank treatment than during the Predator treatment (mixed model: $N=16, F=33.17$, $P<0.0001$; Fig. 1). The increased darting behavior and decreased feeding rates in the Predator treatment period compared to the Blank treatment period showed that the predator model was perceived as a threat.

\section{Experiment 2-size range}

Compared to the eight large rockfish, the eight small rockfish took their first bite earlier (Mann-Whitney test: $N=16, U=51.5, P<0.04$; Fig. 2), and had a nonsignificant tendency to take more bites in the Prepredator treatment period (Mann-Whitney test: $N=16, U=16.5, P=0.098$ ), take more bites in the

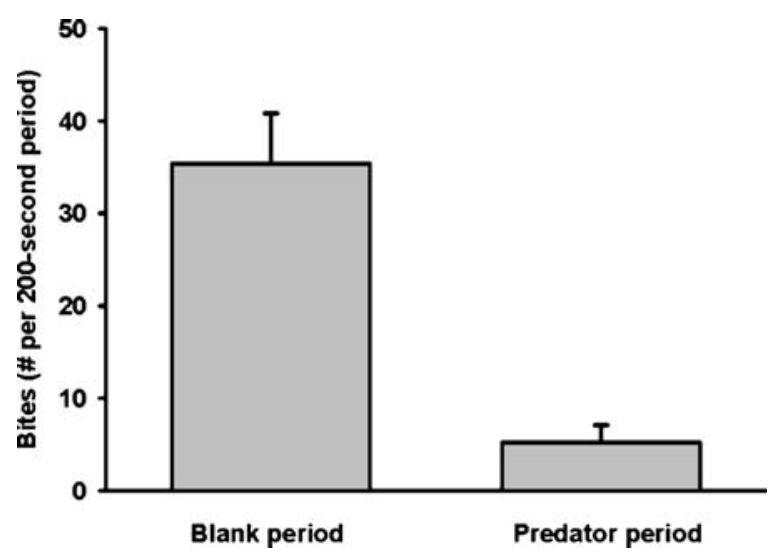

Fig. 1 Test of the predator model. Rockfish took fewer bites when a predator model was present (Predator period) than when no predator model was present (Blank period). The order of Blank and Predator periods was alternated among the 16 individuals. Error bars represent standard errors 


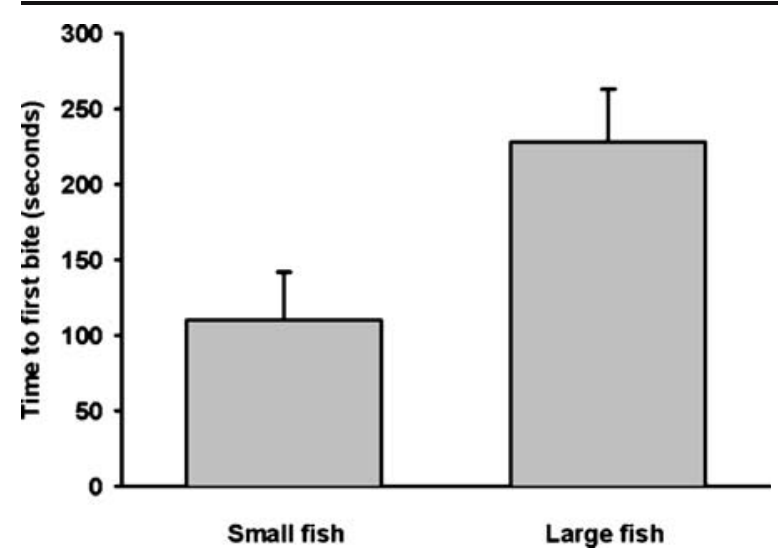

Fig. 2 Effect of body size on behavior. Compared to small fish, large fish $(N=8)$ waited longer before taking their first bites $(N=8)$. Error bars represent standard errors

Predator treatment period (Mann-Whitney test: $N=16$, $U=16.0, P=0.079$ ), and spend more time in the ring surrounding the predator (Mann-Whitney test: $N=16$, $U=6.0, P=0.06$ ).

Experiment 3-behavioral syndromes - standardized body sizes

Two rounds of behavioral assays were conducted on 41 fish. In both rounds 1 and 2, the number of bites taken in the Pre-predator treatment period tended to show a positive correlation with the number of bites taken in the Predator treatment period (Spearman rank correlation: round 1: $r_{\mathrm{s}}=0.30, N=41, P=0.06$; round 2: $r_{\mathrm{s}}=0.55, N=41, P<0.001$; Fig. 3$)$ and with the

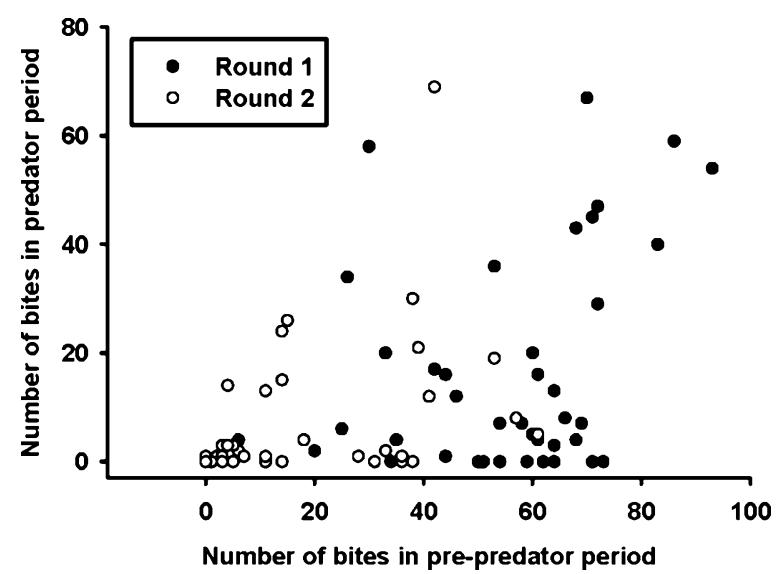

Fig. 3 Correlations between behaviors. Feeding rates in the Pre-predator period predicted feeding rates in the Predator period, in both rounds 1 and 2. Black circles show data from round $1(N=41)$; white circles show data from round $2(N=41)$

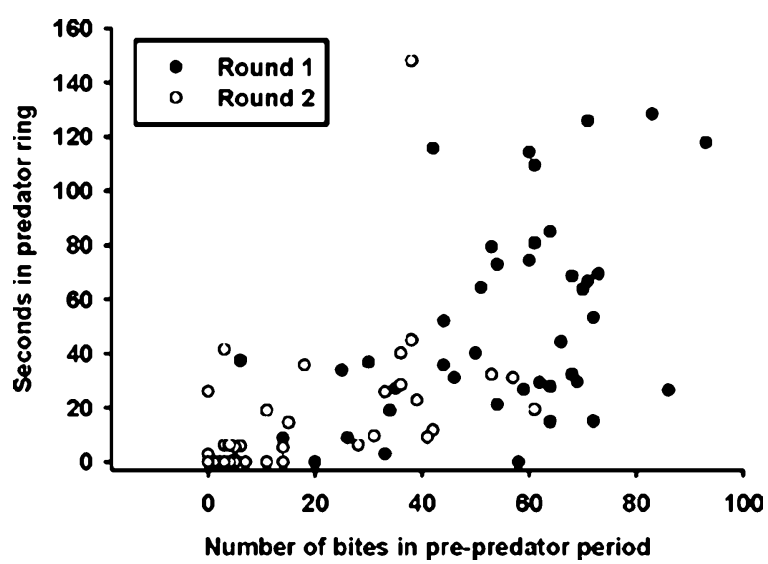

Fig. 4 Correlations between behaviors. Feeding rates in the Pre-predator period predicted time in the predator ring, in both rounds 1 and 2. Black circles show data from round $1(N=41)$; white circles show data from round $2(N=41)$

amount of time spent in the ring surrounding the predator model (round 1: $r_{\mathrm{s}}=0.39, P<0.02$; round 2: $r_{\mathrm{s}}=0.62, P<0.0001$; Fig. 4$)$. The time to the first bite did not correlate with bites taken in the Pre-predator period (round 1: $N=41, P>0.52$; round 2: $N=41$, $P>0.66$ ), the Predator period (round 1: $N=41$, $P>0.81$; round $2: N=41, P>0.77$ ), or the time spent in the ring (round 1: $N=41, P>0.71$; round 2: $N=41$, $P>0.11)$.

Individual behavior in round 1 did not predict behavior in round 2 (Spearman rank correlation: time to first bite: $N=41, P>0.69$, bites in Pre-predator period: $N=41, P>0.30$; bites in Predator period: $N=41$, $P>0.40$; time near the predator model: $N=41, P>0.17$ ). No correlations existed between the relative change in feeding rate between Pre-predator period and Predator period in round 1 versus round $2(N=41, P>0.95)$.

Averaging behavioral values across rounds 1 and 2 for each individual, the four behaviors ranged widely among individuals (means followed by range: time to first bite, $113 \mathrm{~s}, 12$ to $259 \mathrm{~s}$; bites in pre-predator period: 36,7 to 75 ; bites in predator period: 12,0 to 44; time in ring surrounding predator: $33 \mathrm{~s}, 0$ to $92 \mathrm{~s}$ ). This variation suggested that there existed an opportunity to see differences in growth and survival among behaviorally different individuals, if they existed.

Sixteen weeks after the completion of round 2, $78 \%$ (32 of 41) of those fish that had completed round 2 were still alive. None of the behaviors averaged from rounds 1 and 2 predicted whether a fish would survive or die (Mann-Whitney test: time to first bite: 
$P>0.61$; bites in the Pre-predator treatment period: $P>0.94$; bites in the Predator treatment period: $P>0.72$; time spent in the ring near the predator: $P>0.52$ ). Of the surviving fish, growth ranged from 5.5 to $17 \mathrm{~mm}$ and averaged $13.2 \mathrm{~mm}$. Two of the 32 surviving fish (5.5 and $8.0 \mathrm{~mm}$ ) developed deformed jaws during the 16 week rearing period and were therefore excluded from further analyses. However, all of the following statistics are still far from significant when those two data points are included. For those fish that survived the 16 weeks, growth was not predicted by any behavior (Spearman rank correlation: time to first bite: $N=39$, $P>0.14$ for round $1, P>0.81$ for round 2 ; bites in the Pre-predator treatment period: $N=39, P>0.63$ for round 1, $P>0.55$ for round 2; bites in the Predator treatment period: $N=39, P>0.50$ for round $1, P>0.62$ for round 2; time spent in the ring near the predator: $N=39$, $P>0.57$ for round $1, P>0.25$ for round 2). Body size at the beginning of the study did not correlate with body size 16 weeks after round $2(N=39, P>0.36)$.

\section{Discussion}

Behavioral syndromes provide a mechanism through which selection on one character could potentially be amplified to other characters (Huntingford 2004). The data show that behavioral syndromes exist in juvenile brown rockfish. In both rounds 1 and 2 of Experiment 3 , juvenile rockfish that fed at high rates in the absence of a predator also tended to feed at high rates in the presence of the predator model. There was also a correlation in both rounds between feeding rate in the absence of a predator and, after the predator model was added, time near the predator. Behavioral values, averaged across rounds 1 and 2 , ranged widely among individuals. This variation allowed for a test of whether individuals with different behavioral averages survived or grew at different rates in the hatchery. However, no behavior predicted survival or growth in the hatchery.

Why was there no correlation between behavioral traits and success in the hatchery? It is perhaps not surprising that differential growth or survival were not detected among individuals with different behavioral averages, given the instability of individual behavior between the two rounds of Experiment 3, and the condition-dependence demonstrated in Experiment 2. In Experiment 3, behavioral syndromes were stable between rounds 1 and 2, but the behavior of each individual was unstable between those rounds. For example, in both rounds 1 and 2, bites taken in the absence of the predator model correlated with later time near the predator, but an individual's tendency in behavior "a" in round 1 did not predict that individual's tendency for behavior "a" in round 2 . Thus, in contrast with the stable behavioral syndromes, individual behavior at any point in time was ephemeral, apparently reflecting transient variables (e.g. hunger) more than persistent individual personalities. The finding of stable behavioral syndromes but unstable individual behavior is similar to recent work on sticklebacks. Bell and Stamps (2004) recorded three behavioral measures at three ontogenetic stages - juvenile, subadult, and adult, and found a behavioral syndrome that was stable across all three stages, despite the fact that individual behavior was not. Thus stable behavioral syndromes are compatible with unstable (plastic) individual behavior (Bell and Stamps 2004). This current study on brown rockfish complements the stickleback work in showing that behavioral syndrome stability is compatible with behavioral plasticity over an even shorter time period8 to 12 days, compared to the broader ontogenetic stages in the stickleback study.

In Experiment 2, all fish were satiated on Day 1, but small rockfish took their first bite on brine shrimp more quickly than did large rockfish, and tended (marginally non-significantly) to take more bites in the pre-predator and predator periods and spend more time near the predator model. Such condition-dependent risk-taking is predicted by the asset-protection principle, which states that larger individuals should minimize predation risk in order to protect the high expected future fitness that they have already accumulated (Clark 1994). The results are also consistent with the metabolism hypothesis (Brown and Braithwaite 2004), which states that since smaller individuals are at a greater risk of starvation than larger individuals, they should feed more readily and be willing to take greater risks to obtain food.

The plasticity of individual behavior that was discovered in Experiments 3 and 2 may reduce effects of selection on individual behaviors by creating a "moving target" for selection. Results from Experiment 3 suggest that even if voracious feeders grow at high rates, the feeding tendencies of each individual fluctuate through time, making consistent selection on 
particular individuals less likely. Further, the data from Experiment 2 suggest that feeding voracity may decline as fish grow. This condition-dependent feeding behavior could retard or even reverse differential growth among individuals. Behavioral plasticity could also help to dampen effects of selection by restoring variation in a population after selection has occurred (also see Bell and Stamps 2004). At least for the behavioral traits that were measured, at the stage in ontogeny and size range at which the fish were tested, unintentional selection may be less likely to change the behavior of the hatchery population than if the individual behavioral variation were more stable through time.

It would have been ideal to have done these experiments with known multiple family groups (since the number of males that inseminated the mother of the rockfish that were tested is unknown), but brown rockfish stock enhancement is just beginning and such groups were not available. The methods in this study may have underestimated behavioral variation in brown rockfish, however considerable variation was still documented. Further the finding that individuals are plastic in their behavior - the finding that has the greatest implications for hatchery rearing and the hypothesis that this study sought to test - should not change with a greater number of known family groups. Nevertheless, repeating this study with known family groups may lead to additional insights into brown rockfish behavior.

In general, knowledge about behavioral syndromes and behavioral plasticity may allow biologists to determine and prioritize the level and types of potential risk associated with unintentional selection in culture. Species with both stable behavioral syndromes and stable individual behavior may be at greatest risk of amplified effects of unintentional selection in hatcheries (also see Bell and Stamps 2004); the prediction that follows is that species with such behavioral characteristics will be more susceptible to domestication than those species that are less behaviorally stable. Some salmonids contrast with the rockfish used in this study by having not only stable behavioral syndromes, but also stable individual behavior that potentially could be unintentionally selected in culture (Lepage et al. 2000; Huntingford 2004 and references therein, Sundstrom et al. 2004; Brelin et al. 2005; Overli et al. 2005; Schjolden et al. 2005). Salmonids have indeed shown considerable domestication in hatcher- ies (Reisenbichler and Rubin 1999). When both behavioral syndromes and individual behavior are stable, knowledge about the mechanisms through which selection occurs in culture can be used to create culture scenarios to help to ameliorate such selection (Huntingford and Adams 2005). When behavioral traits are unstable, as in the juvenile brown rockfish studied here, the likelihood of unintentional selection may be reduced-even if stable behavioral syndromes exist.

Acknowledgements Mike Rust (NOAA), Ken Massee (NOAA), Tom Waite (University of Idaho), Tim Visi (University of Idaho), and the Seattle Aquarium made it possible for us to obtain and work with the brown rockfish. We thank Alison Bell and Chris Tatara for discussions of the study. Tom Flagg, David Noakes, and three anonymous reviewers provided helpful comments on the manuscript. We are grateful to John Ransier at Northwest Marine Technology for tagging materials, and Jeff Atkins, Skip Tezak, and Abby Tillotson for preparing the flume for the experiments. JSFL was supported by an NIMH training grant during the experiments and by a National Oceanic and Atmospheric Administration (NOAA)-Fisheries funded research consortium-the Science Consortium for Ocean Replenishment (SCORE) during the preparation of the manuscript. The views expressed herein are those of the authors and do not necessarily reflect those of the funding agencies.

\section{References}

Bell AM (2007) Future directions in behavioural syndromes research. Proc R Soc Lond B 274:755-761

Bell AM, Stamps JA (2004) Development of behavioural differences between individuals and populations of sticklebacks, Gasterosteus aculeatus. Anim Behav 68:1339-1348

Bell JD, Bartley DM, Lorenzen K, Loneragan NR (2006) Restocking and stock enhancement of coastal fisheries: potential, problems and progress. Fish Res 80:1-8

Bishop TD, Brown JA (1992) Threat-sensitive foraging by larval threespine sticklebacks (Gasterosteus aculeatus). Behav Ecol Sociobiol 31:133-138

Brelin D, Petersson E, Winberg S (2005) Divergent stress coping styles in juvenile brown trout (Salmo trutta). Ann NY Acad Sci 1040:239-245

Brown C, Braithwaite VA (2004) Size matters: a test of boldness in eight populations of the poeciliid Brachyraphis episcopi. Anim Behav 68:1325-1329

Brown C, Day R (2002) The future of stock enhancements: lessons for hatchery practice for conservation biology. Fish Res 3:79-94

Clark CW (1994) Antipredator behavior and the asset protection principle. Behav Ecol 5:159-170

Cote J, Clobert J (2007) Social personalities influence natal dispersal in a lizard. Proc R Soc Lond B 274:383-390

Duckworth RA (2006) Behavioral correlations across breeding contexts provide a mechanism for a cost of aggression. Behav Ecol 17:1011-1019 
Einum S, Fleming IA (1997) Genetic divergence and interactions in the wild among native, farmed and hybrid Atlantic salmon. J Fish Biol 50:634-651

Fleming IA, Einum S (1997) Experimental tests of genetic divergence of farmed from wild Atlantic salmon due to domestication. ICES J Mar Sci 54:1051-1063

Fleming IA, Petersson E (2001) The ability of released, hatchery Salmonids to breed and contribute to the natural productivity of wild populations. Nord J Freshw Res 75: $71-98$

Huntingford FA (2004) Implications of domestication and rearing conditions for the behaviour of cultivated fishes. J Fish Biol 65:122-142

Huntingford F, Adams C (2005) Behavioural syndromes in farmed fish: implications for production and welfare. Behaviour 142:1207-1221

Lepage O, Overli O, Petersson E, Jarvi T, Winberg S (2000) Differential stress coping in wild and domesticated sea trout. Brain Behav Evol 5:259-268

Levin PS, Holmes EE, Piner KR, Harvey CJ (2006) Shifts in a Pacific ocean fish assemblage: the potential influence of exploitation. Conserv Biol 20:1181-1190

Lima SL, Dill LM (1990) Behavioral decisions made under the risk of predation - a review and prospectus. Can J Zool 68:619-640

Love MS, Yoklavich MM, Thorsteinson L (2002) The rockfishes of the Northeast Pacific. University of California Press, Berkeley, CA, p 405

Lynch M, O'Hely M (2001) Captive breeding and the genetic fitness of natural populations. Conservation Genetics $2: 363-378$

Magnhagen C (2007) Social influence on the correlation between behaviours in young-of-the-year perch. Behav Ecol Sociobiol 61:525-531

Molony BW, Lenanton R, Jackson G, Norriss J (2003) Stock enhancement as a fisheries management tool. Rev Fish Biol Fish 13:409-432
Morgan MJ, Colgan PW (1987) The effects of predator presence and shoal size on foraging in bluntnose minnows, Pimephales notatus. Environ Biol Fishes 20:105-111

Nakagawa M, Okouchi H, Adachi J, Hattori K, Yamashita Y (2006) Effectiveness of stock enhancement of hatcheryreleased black rockfish Sebastes schlegeli in Yamada Bay-evaluation by a fish market survey. Aquaculture 263:295-302

Overli O, Winberg S, Pottinger TG (2005) Behavioral and neuroendocrine correlates of selection for stress responsiveness in rainbow trout - a review. Integr Comp Biol $4: 463-474$

Parker SJ, Berkeley SA, Golden JT, Gunderson DR, Heifetz J, Hixon MA, Larson R, Leaman BM, Love MS, Musick JA, O'Connell VM, Ralston S, Weeks HJ, Yoklavich MM (2000) Management of Pacific rockfish. Fisheries 25:22-30

Reisenbichler RR, Rubin SP (1999) Genetic changes from artificial propagation of Pacific salmon affect the productivity and viability of supplemented populations. Ices J Mar Sci 56:459-466

Salonen A, Peuhkuri N (2006) The effect of captive breeding on aggressive behaviour of European grayling, Thymallus thymallus, in different contexts. Anim Behav 72:819-825

Salvanes AGV, Braithwaite VA (2006) The need to understand the behaviour of fish reared for mariculture or restocking. Ices J Mar Sci 63:346-354

Schjolden J, Stoskhus A, Winberg S (2005) Does individual variation in stress responses and agonistic behavior reflect divergent stress coping strategies in juvenile rainbow trout? Physiol Biochem Zool 78:715-723

Sih A, Bell A, Johnson JC (2004) Behavioral syndromes: an ecological and evolutionary overview. Trends Ecol Evol 19:372-378

Sundstrom LF, Petersson E, Hojesjo J, Johnsson JI, Jarvi T (2004) Hatchery selection promotes boldness in newly hatched brown trout (Salmo trutta): implications for dominance. Behav Ecol 15:192-198 